\title{
A Spiral Nerve Cuff Electrode for Peripheral Nerve Stimulation
}

\author{
GREGORY G. NAPLES, MEMBER, IEEE, J. THOMAS MORTIMER, AVRAM SCHEINER, \\ AND JAMES D. SWEENEY
}

\begin{abstract}
A new type of nerve cuff electrode consisting of conductive segments embedded within a self-curling sheath of biocompatible insulation has been developed. This spiral nerve cuff is biased to selfwrap around peripheral nerves and possesses a "self-sizing" property, presenting an alternative to present commercially available, fixed-size nerve cuffs that are manually wrapped around nerves and sutured shut ("split-cylinder" cuffs). Spiral cuff design and manufacture are described.

We hypothesize that unlike traditional cuffs, the spiral cuff potentially can be implanted safely when sized to fit peripheral nerves snugly. Theoretical pressure analyses of traditional and spiral cuffs that support this hypothesis are presented. These analyses are designed to predict the minimum CNR (cuff diameter/nerve diameter ratio) at which there is no interference with intraneural blood flow. A safe CNR of 0.997 is predicted for a $1 \mathrm{~mm}$ split-cylinder cuff, while a safe CNR of 0.83 is predicted for a $1 \mathrm{~mm}$ spiral cuff. The quantitative relationships between nerve size and safe cuff size are detailed. Results of a preliminary experiment in which snug spiral cuffs were implanted on feline peripheral nerve support the prediction that they may be safe.
\end{abstract}

\section{INTRODUCTION}

A NEW type of nerve cuff electrode for use in neural stimulation and recording has been developed. It consists of electrodes embedded within a self-curling sheath of biocompatible insulation which exhibits a spiral transverse cross section. This spiral nerve cuff electrode was designed to be expandable so that it could be sized to fit snugly around a nerve and/or accommodate neural swelling.

Nerve cuff electrodes are one type of electrode utilized in neuroprosthetic applications such as activation of the lower extremity [9], [29], [30], [57]-[59], the bladder [44], [51], and the diaphragm [20], [21], [25], [26]; treatment of chronic pain [36], [37], [39], [40]; block of neural conduction [50], [54]-[56]; sensory feedback [10]; and recording electroneurograms [12]-[14], [46]-[48]. Among the features that sometimes make nerve cuffs more attractive than other types of electrodes for these appli-

\footnotetext{
Manuscript received November 16, 1987; revised July 10, 1988. This work was supported by the Rehabilitation Research and Development Service of the Veterans Administration, the National Science Foundation, Science and Technology to Aid the Handicapped under Grant PFR80-17190, and the National Institutes of Health-National Institute of Neurological and Communicative Disorders and Stroke, Neural Prostheses Program under Contract N01-NS-3-2344.

The authors are with the Department of Biomedical Engineering, the Applied Neural Control Laboratory, Case Western Reserve University, Cleveland, $\mathrm{OH} 44106$.

IEEE Log Number 8823295.
}

cations are the following:

1) For motor prostheses, muscle length and limb position are expected to have little or no effect on the recruitment characteristic of cuff electrodes, unlike surface and muscle electrodes [11], [22].

2) The stimulus magnitude required for nerve activation is minimized with nerve electrodes, conserving stimulator power and minimizing the likelihood of electrically-induced tissue destruction [32].

3) Cuffs may be positioned so that relative motion caused by muscle contraction and limb movement is minimized. Hence, the probability of lead failure is reduced, resulting in a relatively long lifetime for the electrode.

4) The excitatory field within a cuff theoretically can be accurately controlled, making possible the precise manipulation of current flow and, hence, regulation of the particular neural elements that are activated or blocked [31], [49].

5) The use of nerve cuff electrodes makes viable certain techniques for blocking neural conduction [50], [54][56] and activating motor units in their natural recruitment order [18], [19].

Nerve cuffs are among the most successful of biomedical electrodes. Some have been implanted safely for as long as 15 years [21], [59], a duration unmatched by other neuroprosthetic electrodes. Yet cuffs also have been associated with mechanically-induced neural damage [8], $[25],[26],[29],[30],[37],[39],[40],[47],[59]$. For this reason, the use of nerve cuff electrodes has often been contraindicated. Other factors that also might hinder their use include the present difficulty of precisely grading the force output of target muscles (due to the very steep recruitment curves exhibited by cuffs) and the difficulty of removing the electrode if an explant procedure becomes necessary.

Numerous variations of the basic nerve cuff design have been described in the literature, many of which do not appear to be practical for long-term human implantation because of their mechanical or geometrical properties. ${ }^{1}$ Designs by McCarty [27], [28] and Sauter et al. [43] appear to require a potentially traumatic implantation procedure. Designs by Barone et al. [5] and Dubkin [15] also are potentially traumatic as each consists of a stiff seg-

'It should be noted that in many of these reports the electrodes were not portrayed as necessarily being suitable for use in humans. 
ment of tubing that is clasped to the nerve with a flexible piece of rubber. Designs by Banzett [4], Ninomiya et al. [38], and Schwartz et al. [45], [52] appear to be overly bulky and inflexible compared to the nerve tissue. Designs by Julien and Rossignol [24] and Testerman [53] are bonded directly to the nerve and are appropriate only for acute recording purposes. Designs by DeLuca et al. [14] and Edell [17] may be safe for long-term implantation but are specially designed for recording from severed nerves.

Thus only a few configurations, characterized by electrodes set within soft, flexible sheaths of insulation, are truly appropriate for clinical implementation as neuroprosthetic components. These are the "split-cylinder" cuffs (see Fig. 1) which are installed by being wrapped around the nerve and secured with suture, thus forming a tube that encompasses the nerve [2], [3], [23]. ${ }^{2}$ These commercially available designs have commonly been used in neural stimulation and recording systems for long-term implantation on peripheral nerve.

Despite both the functional success of the various clinical applications in which these cuffs have been utilized, and the longevity of many of the implants, their use has not become widespread, principally because they also have been associated with the occasional infliction of neural damage. Several studies describe evaluations of particular neuroprostheses employing nerve cuffs and anecdotally note the sporadic occurrence of neural damage. These include Nielson et al. [37] and Picaza et al. [39], [40] for pain relief systems; Breederveld and Zilvold [8], Waters et al. [57]-[59], and McNeal et al. [29], [30] for foot-drop assist devices; Yuen et al. [60] for a micturition assist device; and $\mathrm{Kim}$ et al. [25], [26] for a respiratory assist device. In addition, Stein et al. [47] describe damage inflicted by recording cuffs.

Various forms of neural damage are described by these authors: loss of function of the innervated muscles, increased amounts of intraneural connective tissue, the presence of cells associated with the foreign body response of the immune system, Wallerian degeneration, demyelination, and a decrease in the population of larger axons. Direct mechanical interaction between cuff and nerve is an obvious means by which such damage might be inflicted; however, there are other mechanisms that also may contribute. Among these are: 1) surgical trauma to either the nerve itself or its blood supply; 2) pressure caused by either seroma formation or excessive fibrous encapsulation around the cuff; 3) the transmission of forces from adjacent muscles to the cuff and, hence, to the nerve; and 4) undue tension in the cuff's leads if they were not carefully routed during implantation.

For the cuff electrode itself, important factors relating to safety include chemical composition, mechanical flexibility, geometric configuration, and size. Accepted biomaterials such as medical-grade Silastic ${ }^{\circledR}$ and Teflon ${ }^{\circledR}$, type 316 stainless steel, and platinum should be used in

${ }^{2}$ The very flexible helical nerve electrode [51], [60], developed at the Huntington Medical Research Institutes, also deserves mention as potentially being safe for clinical application, though it is not really a cuff per se.
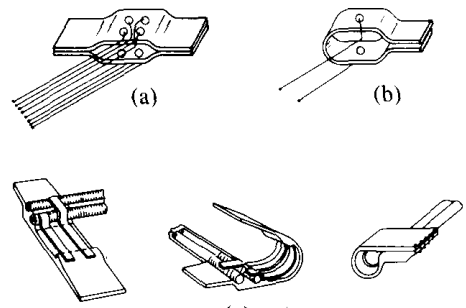

(c)

Fig. 1. The most extensively used of the previously available nerve cuff electrodes have been those of (a) Avery and Wepsic [2], (b) Avery [3] and (c) Hagfors [23]. These "split-cylinder" designs are characterized by electrodes set within flexible pieces of biocompatible insulation that are wrapped around the nerve and sutured shut to secure them.

cuff manufacture. The cuff's mass should be minimized and its flexibility maximized. In addition, cuffs should be made as small and smooth as possible; the exception being that, in order to avoid nerve constriction, cuff diameter typically has not been minimized. It is this parameter, cuff diameter, that commands the most attention with regard to passively-induced neural damage.

The Association for the Advancement of Medical Instrumentation (AAMI) American National Standard for Implantable Peripheral Nerve Stimulators [1] recommends that to be safe for implantation, cuff electrode diameter should exceed nerve diameter by at least 50 percent. This advice is based upon "clinical experience and laboratory studies." The work of Ducker and Hayes [16] is cited specifically to justify the recommendation. Unfortunately, this is somewhat misleading since their research deals not with cuff electrodes implanted on intact nerves, but rather with "regeneration"' cuffs used to improve neural regeneration at an anastomosis. Ducker and Hayes [16] investigated the use of nerve cuffs to improve peripheral nerve repair in dogs and chimpanzees. They concluded that in man the ideal cuff "necessary to achieve maximal neural direct spanning at the site of repair" without connective tissue or neuroma buildup would be as thin as possible, have an internal cross-section area 2.53 times that of the nerve and have a length sufficient to cover the anastomosis without fear of slipping away from it. Thus, their recommendation for optimally-designed human cuffs, based on the chimpanzee implants, was that cuff diameter exceed nerve diameter by $60-70$ percent. In the dogs, optimal cuff diameter exceeded nerve diameter by 40 percent. It is unclear how the AAMI arrived at its 50 percent recommendation.

While these results have some relevance to the safety of nerve cuffs, the study cannot be considered to provide definitive evidence concerning how to size cuff electrodes on intact peripheral nerves. "Regeneration" cuffs must be designed so that the postneurotmesis edema and neuroma formation that commonly accompany nerve transection are taken into account. Stimulating cuffs that are implanted on intact nerves need to satisfy such criteria only minimally. In addition, cuff dimensions that do not result in "optimal axonal spanning" in nerve repair cannot necessarily be assumed to be unsafe for a nerve cuff elec- 
trode. The optimal dimensions of cuff electrodes thus could very well be different than those of regeneration cuffs.

Results of the clinical evaluations cited earlier support to some extent the present recommendation that cuff diameter considerably exceed nerve diameter. However, the issue of safe and unsafe nerve cuff diameters remains open to debate since none of those studies has addressed, in a comprehensive manner, the determination of the precise values of cuff diameter at which safety is compromised. We make these observations with the expectation that revision of the AAMI standard may be required in the future. Until such revision is forthcoming, investigators should continue to follow the AAMI recommendation when traditional cuffs are utilized since it presently represents the best available standard for those designs.

Our new spiral cuff was designed with the expectation that it might be safe for implantation at sizes previously considered to be hazardous. In fact, we believe that the spiral cuff may be safely implanted when sized to fit peripheral nerve snugly. This hypothesis is supported by: 1) the results of a theoretical pressure analysis of the spiral cuff design; and 2) the results of a preliminary experiment in which spiral cuffs were implanted on feline peripheral nerve. A comparative pressure analysis of traditional splitcylinder nerve cuffs confirms the expectation that they cannot be implanted snugly.

\section{Spiral Cuff Design and Manufacture}

The spiral nerve cuff electrode (see Fig. 2) was designed to be easy to install on peripheral nerve without the use of sutures to secure it and also to have the potential of being safe for implantation when sized to be in intimate contact with the nerve [33], [34]. The spiral geometry of the cuff was developed so that it would fully encompass the nerve and yet would be free to expand if it were slightly smaller than the nerve or if swelling were to occur. Such expansion cannot occur if a cuff is sutured shut.

The cuff is manufactured by bonding together two sheets of flexible, biocompatible insulation of different lengths with electrodes sandwiched between them (see Fig. 3). One sheet is clamped at both ends, stretched, and fixed in the stretched position. Electrodes are placed on the stretched sheet; an adhesive layer is spread over the electrodes and the stretched sheet; a second unstretched sheet of material is placed on top of the adhesive; and the composite is compressed to a constant thickness. After the adhesive sets, windows are cut into the stretched sheet to expose the electrodes to the interior surface of the cuff. The cuff itself is then cut from the bonded sheets and trimmed. The cuff, having been released, coils into a tube with a spiral transverse cross section as the stretched sheet contracts to its natural rest length while the unstretched sheet remains at its rest length. The cuff's diameter is related to the amount of stretch: the greater the stretch, the tighter the cuff will curl. A cuff can consist of as many wraps as desired.

The electrodes sandwiched between the sheets are pres-

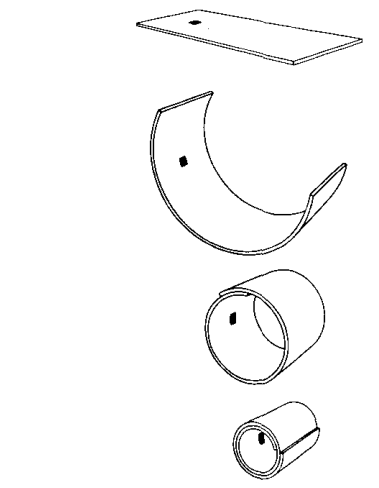

(a)

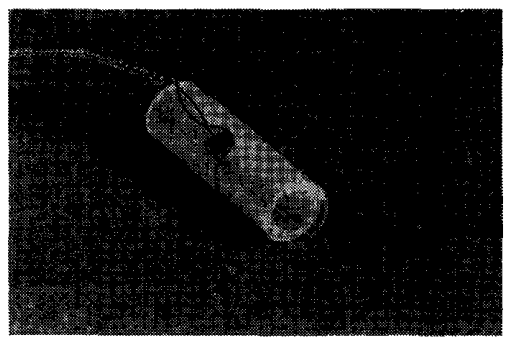

(b)

Fig. 2. The spiral nerve cuff electrode was designed so that it could be easily implanted on peripheral nerve without the need of suturing to secure it there. (a) Shown in this diagram are, from top to bottom: the planar structure in the fully opened (or stretched) state; a partially opened (or stretched) state where the cuff could accept a peripheral nerve; a partially closed state such as that which would exist if the cuff were implanted on a nerve that had a diameter greater than that of the cuff, either naturally or due to swelling; and finally, the fully closed state in which the cuff exhibits its natural resting diameter. (b) An actual monopolar spiral nerve cuff electrode. The cuff has a length of about 20 $\mathrm{mm}$, an internal diameter of about $4 \mathrm{~mm}$, and a wrap thickness of about $0.3 \mathrm{~mm}$.

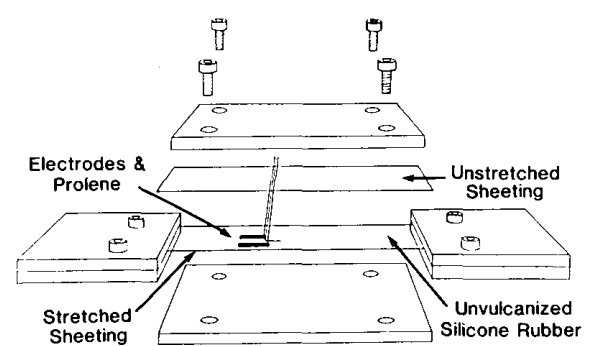

Fig. 3. Spiral cuffs are manufactured by bonding together two pieces of silicone rubber sheeting having different natural resting lengths. The shorter piece is fixed in a stretch state until it can be bonded to the longer piece. This procedure is detailed in the text.

ently made from thin, biocompatible metal foil with geometries ranging from small buttons to circumneural bands. Each electrode is spot-welded to a length of lead wire. During manufacture the electrodes are laid upon the stretched sheet according to a desired predetermined geometry. The lead wires extend out in a direction perpendicular to that of the stretch.

"Blocking" cuff geometries (see Fig. 4) like that introduced by Sweeney and Mortimer [50] also can be created 


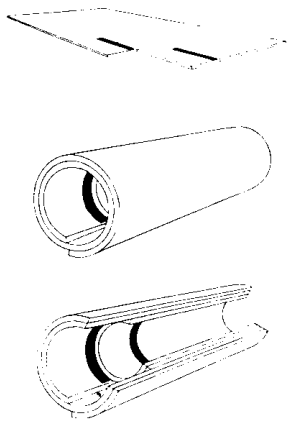

Fig. 4. Blocking cuffs like those described by Sweeney and Mortimer [50] can be created easily by appropriately trimming the cuff.

via this technique. A bipolar cuff electrode with circumneural electrodes is used in this application. The blocking geometry differs from standard stimulating and recording geometries in that the anode and cathode have different diameters. Such a geometry can be created simply by cutting a rectangular segment from one corner of an unrolled spiral cuff. When allowed to roll up once again, the cuff will exhibit two internal regions with different diameters.

A detailed example of the construction of a monopolar cuff with a button electrode is presented here to illustrate the manufacturing technique. First, a piece of 99.9 percent pure platinum foil (the electrode) having an area of $4.0 \mathrm{~mm}^{2}$ and a thickness of $25 \mu \mathrm{m}(0.001 \mathrm{in})$ is spotwelded to a length of Teflon ${ }^{\circledR}$-insulated type 316 multistrand stainless steel wire (Cooner Wire Co.), $2 \mathrm{~mm}$ of which has been deinsulated. A second wire may also be welded to the foil if redundant leads are desired. Next, a piece of Silastic ${ }^{\circledR}$ sheeting (Dow Corning Co.) having a thickness of $127 \mu \mathrm{m}$ ( 0.005 in) is clamped at both ends, stretched, and secured at a predetermined length according to the desired cuff diameter (details presented below). The electrode is positioned appropriately on top of the stretched sheet, alongside a length of 5-0 Prolene ${ }^{\circledR}$ (Ethicon, Inc.). For cuffs containing multiple electrodes, extreme care must be taken to position the electrodes accurately according to a previously determined desired geometry. The lead wire and Prolene ${ }^{\circledR}$ are oriented in a direction perpendicular to that of the stretch of the sheet. A thin layer of Dow Corning ${ }^{\circledR}$ MDX-4-4210 Clean Grade Elastomer is spread on top of the sheeting and electrode, and a piece of unstretched sheeting is then placed on top of the MDX-4-4210. The composite is compressed to a constant thickness of $300 \mu \mathrm{m}$ and heated until the MDX, a silicone elastomer purchased in an unvulcanized state, has cured, thus bonding the two pieces of sheeting together. After cooling, the composite is removed, a window is cut to expose the electrode to the cuff's interior, and the cuff is cut out and trimmed according to the desired dimensions. Typically, a cuff would be $7-20 \mathrm{~mm}$ in length and consist of one and a half to two wraps. Finally, the lead is wound into a helical coil with a bore diameter of $225 \mu \mathrm{m}$ and the Prolene ${ }^{\circledR}$ is fed through the length of the coil. The end of the lead wire is deinsulated $(5 \mathrm{~mm})$ and crimped, together with the Prolene ${ }^{\circledR}$, within a small piece of stainless steel tubing which serves as the connector for the electrode. The Prolene ${ }^{\circledR}$ core, secured at both ends, prevents the coiled lead from unraveling.

\section{Stretch-Diameter Relationship}

It is possible to relate the amount of stretch applied to the first Silastic ${ }^{\circledR}$ sheet and the diameter of the resulting cuff. For this analysis it is assumed that once the two sheets are bonded and released, the stretched sheet contracts back to its original resting length and the unstretched sheet remains at its original resting length. ${ }^{3}$ Thus, the inner surface of the cuff (which, from Fig. 3, is seen to correspond to the unbonded surface of the stretched piece of sheeting) will have a circumference equal to the natural resting length of the sheet which had been stretched, while the outer surface of the cuff (corresponding to the unbonded surface of the unstretched piece of sheeting) has a circumference equal to the natural resting length of the unstretched sheet. It also is assumed that the thickness and composition of the two sheets are identical so that their properties are the same.

Consider a single wrap cuff (i.e., a cuff that curls through $360^{\circ}$ only once) consisting of two bonded pieces of silicone rubber sheeting without embedded conductors. The diameter of the inner surface of the cuff is $D_{1}$ and that of the outer surface is $D_{2}$. The circumference of the inner surface (corresponding to the resting length of the stretched sheet) is $X_{1}$ and that of the outer surface (corresponding to the resting length of the unstretched sheet) is $X_{2}$. Let $P$ be the stretch applied to the first sheet expressed as a percentage of its rest length (e.g., 20 percent). Let $T$ be overall cuff thickness.

Equations (1)-(4) below hold true; the first being a mathematical identity. Equations (5)-(7) are then simply derived.

$$
\begin{aligned}
X_{2} & =X_{1} \times\left\{1+\left[\left(X_{2}-X_{1}\right) / X_{1}\right]\right\} \\
X_{1} & =\pi D_{1} \text { and } X_{2}=\pi D_{2} \\
D_{2} & =D_{1}+2 T \\
P / 100 \% & =\left[\left(X_{2}-X_{1}\right) / X_{1}\right] \\
X_{2} & =X_{1} \times\left\{1+\left[\left(D_{2}-D_{1}\right) / D_{1}\right]\right\} \\
& =X_{1} \times\left\{1+\left[2 T / D_{1}\right]\right\} \\
X_{2} & =X_{1} \times\{1+[P / 100 \%]\} \\
P / 100 \% & =2 T / D_{1} .
\end{aligned}
$$

Hence, percent stretch and cuff diameter are seen to be simply related

${ }^{3}$ Actually, it is assumed only that the unbonded surfaces of each sheet attain their natural resting lengths. Clearly, neither sheet exhibits its resting length throughout its entire thickness since, at the boundary between the two sheets, the bonded surface of the stretched sheet will be held in tension as the unstretched sheet tends to hold it in its stretched state, and the bonded surface of the unstretched sheet will be "pulled" into compression as the stretched sheet tends to contract. This gives rise to a complex, varying strain profile throughout the thickness of the cuff. An exact analysis that takes such a profile into account is beyond the scope of this paper. 


$$
\begin{gathered}
P=100 \% \times\left(2 T / D_{1}\right) \\
D_{1}=100 \% \times(2 T / P) .
\end{gathered}
$$

A simple geometric analysis thus yields a first-order estimate of the stretch-diameter relationship associated with the spiral cuff.

These relationships hold true for single wrap cuffs without embedded electrodes. For multiple wrap cuffs, the amount of stretch required to obtain a specific cuff diameter, or the cuff diameter resulting from a specific amount of stretch, will actually be less than that predicted by (8) and (9). Each turn of the cuff will tend toward the diameter predicted by the equations. Therefore, the innermost wrap of a multiple wrap cuff will be compressed to a smaller diameter by the successive outer wrap(s). There is a limit to this behavior at the point where friction between successive wraps coupled with the natural tendency for the inner wrap(s) to expand to the diameter predicted by the equations balances the compression exerted by the outer wrap(s). Observations indicate that cuffs with one and a half or more wraps, immersed in a physiological medium (to approximate the lubrication to which an actual implant would be exposed), will exhibit diameters approximately 10 percent smaller than those of single wrap cuffs made at the same degree of stretch. Therefore, the equations for multiple wrap cuffs can be approximated as

$$
\begin{aligned}
P & =0.9 \times\left[100 \% \times\left(2 T / D_{1}\right)\right] \\
D_{1} & =0.9 \times[100 \% \times(2 T / P)] .
\end{aligned}
$$

The relationships developed here provide a fairly accurate guide to cuff manufacture as long as care is exercised during the process; however, variations are not uncommon. For example, we have observed that actual single wrap and multiple wrap cuffs often are somewhat larger than (9) and (11), respectively, predict. This deviation most likely is due to the fact that the adhesive layer bonding the two sheets together behaves in the same manner as the unstretched sheet, tending to hold the cuff open, i.e., it effectively increases the thickness of the unstretched sheet; hence, the cuffs do not tend to curl as tightly as they would if the stretched and unstretched layers had identical thicknesses. ${ }^{4}$ It should be possible to develop a more accurate stretch-diameter relationship for the spiral cuff, one that would incorporate the basic geometrical characteristics considered here, the added effects of the bonding layer, the mechanical properties of the materials used, variations in geometry resulting from the applied loads, and other factors as well. Such an analysis is beyond the scope of this discussion however.

It must be appreciated that while (8)-(11) provide general guidelines for manufacturing a spiral cuff of a particular size, the inclusion of conductive segments to serve

\footnotetext{
${ }^{4}$ Variations in cuff diameter also might be caused by variations in the thickness of either the sheeting or the bonding layer, variations in sheeting width, slippage of the sheeting where it is clamped, distortion of the sheeting as it is stretched, viscoelasticity of the sheeting, and measurement errors.
}

as electrodes can alter its final diameter since the stiffness of the electrodes may be great enough to hold the cuff at a size different from that predicted by the equations. Observations indicate that a cuff's diameter may be altered by as much as 20 percent of its natural resting diameter, depending on the number, size, and mechanical properties of the electrodes. For example, very little alteration is possible if the conductive segment is a single very small button, while considerable alteration is possible if the conductors form two or three circumneural bands.

\section{Pressure Analyses for Nerve Cuffs}

In order to evaluate the hypothesis that the spiral cuff will be safe for snug implantation on peripheral nerve, a simplified mechanical analysis was developed to calculate the pressure induced on a nerve by such a cuff. A similar analysis for split-cylinder cuffs was developed for comparative purposes. Each analysis is based on the specific geometrical characteristics of the respective cuffs. In what follows, these analyses are used to demonstrate simply the conditions necessary for safe implantation of snug nerve cuffs of both the split-cylinder and spiral geometries.

The possible effects of electrodes and leads are not accounted for in these analyses. Therefore, they reflect only partial evaluations of actual cuff electrode assemblies. It is assumed that leads would play a minimal role in the infliction of neural damage if care is taken at implantation to route them so that they do not contact the nerve and so that they are left with enough slack that tension would not be transmitted to the cuff. Other assumptions made for these analyses include the following:

1) Young's modulus for the Silastic ${ }^{\circledR}$ cuffs is assumed to be constant. (Young's modulus does vary with the stress applied to the material but is essentially constant within the range we deal with here.)

2) Deflections are assumed to be small, so that the geometries of the systems do not change significantly with the applied loads.

3) The nerve segment within the cuff is treated as an incompressible fluid within a fixed volume.

Consider the implantation of a "snug" cuff upon a nerve. Since the nerve tissue is modeled as an incompressible fluid, it is free to conform to the circular cross-sectional geometry of the cuff. ${ }^{5}$ However, the cross-sectional area of the nerve cannot change. Therefore, the cuff will have to stretch or open in order to accommodate nerves larger than itself. As a result, the elastic restoring forces in the cuff give rise to an increase in the pressure within the enclosed nerve "fluid." Such a pressure increase is presumed to be the underlying mechanism through which damage is inflicted, doing so indirectly by occluding blood flow in the neural microvasculature.

It is important to note that modeling the nerve tissue within the cuff as a fixed volume of incompressible fluid is a "worst case" assumption. If the actual nerve tissue

${ }^{5}$ We have noted, as have others (W.F. Agnew, personal communica-
ion), that this does occur, even when nerves are implanted with loose nerve tion), that this does occur, even when
cuffs (unpublished observations). 
is in fact compressible, or if the volume can change due to longitudinal fluid flow, the actual pressure changes will be smaller than those calculated below.

In what follows, our primary goal is to predict the value of CNR (cuff/nerve diameter ratio) at which interference with intraneural blood flow will begin for each cuff design. The CNR value so predicted will represent the minimum allowable ratio of cuff to nerve diameter for a given cuff size. According to Rydevik et al. [41], interference with intraneural blood flow can first be noted with the application of $20 \mathrm{~mm} \mathrm{Hg}$ of pressure to a peripheral nerve. In the analyses that follow, the CNR values that produce a $20 \mathrm{~mm} \mathrm{Hg}$ increase in internal cuff pressure for various cuff diameters will be calculated. This is a "worst case" condition since only minimal interference with intraneural blood flow can be detected at this point. Clearly, greater pressures (smaller CNR values) result in greater interference with blood circulation.

\section{Split-Cylinder Nerve Cuffs.}

This type of cuff is modeled as an open-ended thickwalled cylinder (Fig. 5). It has been shown [7] that for this geometry, the relationship between the change in internal radius and the change in internal pressure is

$$
\begin{aligned}
\Delta r= & \frac{r_{c}^{3} \Delta P}{E\left[\left(r_{c}+h\right)^{2}-r_{c}^{2}\right]} \\
& *\left[(1-v)+(1+v) * \frac{\left(r_{c}+h\right)^{2}}{r_{c}^{2}}\right] \\
\Delta r & =\text { Change in Internal Radius } \\
\Delta P & =\text { Change in Internal Pressure } \\
r_{c} & =\text { Internal Cuff Radius } \\
h & =\text { Cuff Wall Thickness } \\
E & =\text { Young's Modulus for Silastic } \\
v & =\text { Poisson's Ratio for Silastic. }
\end{aligned}
$$

If a cuff with an original internal diameter of $2 r_{c}$ is placed on a nerve with a diameter of $\left(2 r_{c}+2 \Delta r\right)$, a pressure of $\Delta P$ will be produced.

Given a particular cuff size, we would like to know the size of the nerve that can be implanted with a cuff such that an internal pressure increase of $20 \mathrm{~mm} \mathrm{Hg}\left(1 \mathrm{~N} / \mathrm{mm}^{2}\right.$ $=7500 \mathrm{~mm} \mathrm{Hg}$ ) will be produced. We are also interested in the corresponding CNR for that nerve/cuff pair. Cuffs typically have a wall thickness of $0.3 \mathrm{~mm}$. For a $1 \mathrm{~mm}$ diameter cuff the internal cuff radius is $0.5 \mathrm{~mm}$. Young's modulus and Poisson's ratio for typical Silastic ${ }^{\circledR}$ are $E=$ $2.5 \mathrm{~N} / \mathrm{mm}^{2}$ and $v=0.5$, respectively.

Substituting these values into (12), we obtain

The size of the nerve is

$$
\Delta r=1.48 \times 10^{-3} \mathrm{~mm} \text {. }
$$

$$
\left(2 r_{c}+2 \Delta r\right)=1.003 \mathrm{~mm} \text {. }
$$

The CNR is equal to natural cuff diameter divided by nerve diameter
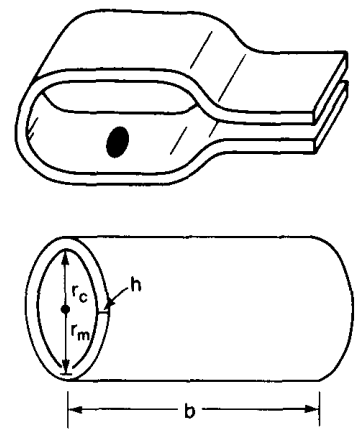

Fig. 5. Split-cylinder nerve cuffs, like that developed by Avery [3], may be modeled as open-ended, thick-walled cylinders. Internal cuff radius $=r_{c}$; mean tube radius $=r_{m}$; tube thickness $=h$; tube length $=b$. The details of the pressure analysis are found in the text.

$$
\mathrm{CNR}=1.0 / 1.003=0.997 .
$$

Thus, if a $1 \mathrm{~mm}$ split-cylinder cuff is placed on a nerve with a diameter of $1.003 \mathrm{~mm}$, internal pressure will increase by $20 \mathrm{~mm} \mathrm{Hg}$.

According to this analysis, if a $1 \mathrm{~mm}$ split-cylinder cuff is placed on a nerve with a diameter more than 0.3 percent larger than that of the cuff, interference with intraneural blood flow may occur. Similarly, if a nerve with a diameter of $1 \mathrm{~mm}$ or less were implanted with a $1 \mathrm{~mm}$ cuff, and if the nerve were to swell to a diameter that was more than 0.3 percent greater than that of the cuff, interference with blood flow might occur. The situation improves for nerves larger than $1 \mathrm{~mm}$ in diameter, but not by much. Illustrated in Fig. 6 is the relationship between nerve size and the minimum allowable split-cylinder cuff CNR so as to avoid interference with intraneural blood flow. As shown in the figure, the CNR causing blood flow interference for a $10 \mathrm{~mm}$ cuff would be 0.98 and for a $20 \mathrm{~mm}$ cuff would be 0.96 . This analysis demonstrates that it would be virtually impossible to accurately size a splitcylinder cuff onto a nerve so that it would be nonconstrictively snug.

\section{B. Spiral Nerve Cuff}

For this analysis, consider a two-wrap spiral cuff without friction between the layers. This assumes that the moistness of the physiological medium provides lubrication for the implanted cuff. This spiral cuff is modeled as two overlapping "snap rings" (Fig. 7). For a single snap ring it can be shown [6] that a change in the gap distance of the ring is related to a change in internal pressure by

$$
\Delta Y=\frac{2.25 \pi D^{4} \Delta P}{E h^{3}}
$$

$\Delta Y=$ Change in Gap Distance

$\Delta P=$ Change in Internal Pressure

$D=$ Mean Snap Ring Diameter

$h=$ Snap Ring Thickness

$E=$ Young's Modulus. 


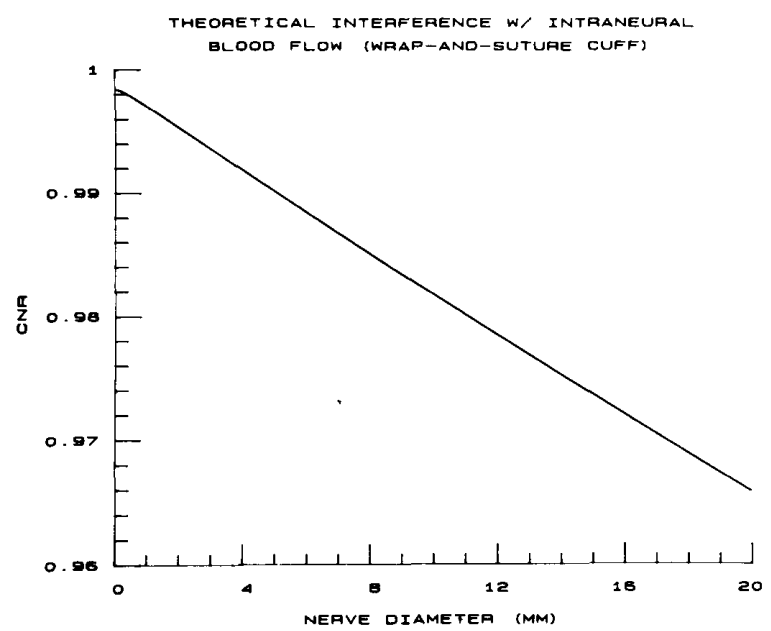

Fig. 6. Theoretical relationship between nerve size and the minimum allowable split-cylinder cuff CNR (cuff/nerve diameter ratio) necessary to avoid interference with intraneural blood flow.
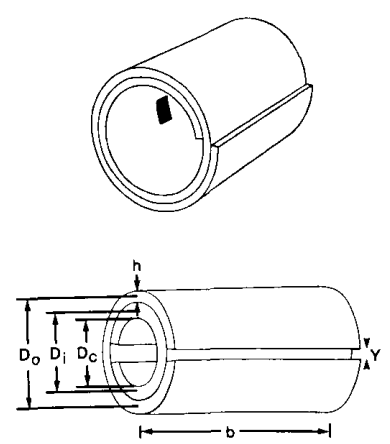

Fig. 7. A two-wrap spiral nerve cuff may be modeled as two overlapping snap rings. Internal cuff diameter $=D_{c} ;$ mean inner ring diameter $=D_{i}$; mean outer ring diameter $=D_{o}$; gap distance $=Y$; ring thickness $=h$; ring length $=b$. The details of the pressure analysis are found in the text.

Assuming that the snap ring remains circular, the corresponding change in ring diameter is

$$
\Delta D=\frac{\Delta Y}{\pi}=\frac{2.25 D^{4} \Delta P}{E h^{3}} .
$$

The pressure needed to cause a given change in snap ring diameter is thus

$$
\Delta P=\frac{E h^{3} \Delta D}{2.25 D^{4}}
$$

For two snap rings (Fig. 7) the internal pressure needed to cause a given change in ring diameter is

$$
\Delta P=\frac{E h^{3} \Delta D}{2.25 D_{i}^{4}}+\frac{E h^{3} \Delta D}{2.25 D_{o}^{4}}
$$

where $D_{i}$ is the mean diameter of the inner snap ring and $D_{o}$ is the mean diameter of the outer snap ring.
The change in diameter is the same for both rings, and they have the same thickness and are composed of the same material. Therefore, (16) can be rewritten as

$$
\Delta P=\frac{E h^{3} \Delta D}{2.25} *\left(\frac{1}{D_{i}^{4}}+\frac{1}{D_{o}^{4}}\right)
$$

The change in diameter, given a change in internal pressure, is thus

$$
\Delta D=\frac{\frac{2.25 \Delta P}{E h^{3}}}{\left(\frac{1}{D_{i}^{4}}+\frac{1}{D_{o}^{4}}\right)} .
$$

We will again determine the size of the nerve that can be implanted with a $1 \mathrm{~mm}$ spiral cuff such that a $20 \mathrm{~mm}$ $\mathrm{Hg}\left(1 \mathrm{~N} / \mathrm{mm}^{2}=7500 \mathrm{~mm} \mathrm{Hg}\right)$ increase in internal pressure will be produced. The corresponding CNR will be calculated as well. Note that for this cuff, $D_{c}=1.0 \mathrm{~mm}$, $D_{i}=1.3 \mathrm{~mm}$, and $D_{o}=1.9 \mathrm{~mm}$. The values for cuff wall thickness $(h=0.3 \mathrm{~mm})$ and Young's modulus $(E=2.5$ $\mathrm{N} / \mathrm{mm}^{2}$ ) are unchanged from the previous example.

Substituting these values into (18), we obtain

$$
\Delta D=0.21 \mathrm{~mm} \text {. }
$$

The size of the nerve is

$$
\left(D_{c}+\Delta D\right)=1.21 \mathrm{~mm} \text {. }
$$

The CNR is equal to natural cuff diameter divided by nerve diameter

$$
\mathrm{CNR}=1.0 / 1.21=0.83 .
$$

Thus, if a $1 \mathrm{~mm}$ spiral cuff is placed on a nerve with a diameter of $1.21 \mathrm{~mm}$, it will induce a $20 \mathrm{~mm} \mathrm{Hg}$ increase in internal pressure.

According to this analysis, a $1 \mathrm{~mm}$ diameter spiral cuff can be safely implanted on nerves up to $1.21 \mathrm{~mm}$ in diameter (up to 21 percent larger than the cuff) without occluding intraneural blood flow. Similarly, if a nerve with a diameter of $1 \mathrm{~mm}$ or less were implanted with a $1 \mathrm{~mm}$ spiral cuff, it could safely swell to a diameter of $1.21 \mathrm{~mm}$ (a 21 percent increase) before any interference with intraneural blood flow would be expected to occur. For larger nerves and cuffs the situation improves. Illustrated in Fig. 8 is the relationship between nerve size and the minimum allowable spiral cuff CNR so as to avoid interference with intraneural blood flow. Note that it is possible to implant a nerve with a cuff that is snug (i.e., CNR $<1.0$ ) but that still has a CNR value greater than the minimum allowable. It thus is possible to implant a cuff snugly and at the same time allow for some postoperative swelling. The amount of swelling that can be accommodated depends upon both initial nerve size and initial implant CNR.

It is appropriate to consider briefly the possible influence that friction between the wraps of a spiral cuff might have on these results, although a quantitative analysis that includes these effects is beyond the scope of this paper. 


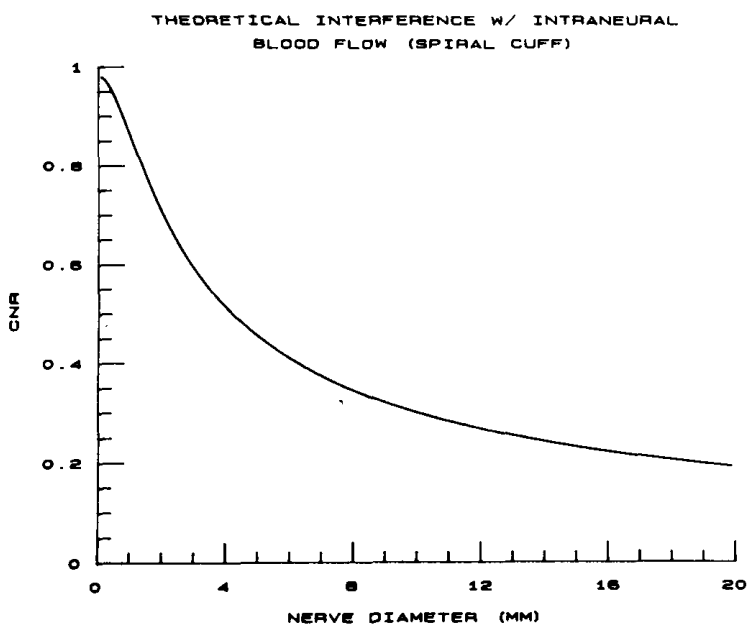

Fig. 8. Theoretical relationship between nerve size and the minimum allowable spiral cuff CNR (cuff/nerve diameter ratio) necessary to avoid interference with intraneural blood flow.

Frictional effects can have either positive or negative consequences, depending on the particular circumstances. Consider the case of snugly implanting spiral cuffs with the expectation that significant neural swelling will not occur. The effects of friction then would be such that the safety of the cuff is more pronounced than that presented above since friction would tend to prevent the outer wrap from squeezing down on the inner wrap and the nerve (i.e., a component of the compressive force exerted by the outer wrap would be offset by the friction between the two wraps). On the other hand, consider the case in which there is an expectation that significant neural swelling will occur. The effects of friction then would be such that the safety of the cuff is less pronounced than that presented above since friction would tend to make it more difficult for the cuff to open and close in response to such swelling.

The results of the spiral cuff pressure analysis are promising, but is the spiral nerve cuff truly safer than splitcylinder cuffs for long-term implantation? A thoroughly assessed series of animal implants, employing both designs, is required to answer this question. Such evaluations are planned for the future. A preliminary implant evaluation of the spiral cuff, serving as a prototype experiment, is described in the following section.

\section{Passive Implant Evaluation}

In order to test the hypothesis that the spiral cuff could be safely implanted on peripheral nerve when sized to fit snugly, a preliminary implant evaluation was performed. Fifty spiral cuffs of various sizes without electrodes or leads were manufactured, subjected to ethylene oxide sterilization, and made available for aseptic implantation on the ulnar, median and sciatic nerves of an adult cat for seven months. Of the six cuffs actually used, four were implanted on the ulnar and sciatic nerves snugly (i.e., the CNR was 0.8-0.9), and two were implanted on the me- dian nerves so that they were slightly loose (i.e., the CNR was about 1.2$).{ }^{6}$ All cuffs consisted of two wraps and were $10.0 \mathrm{~mm}$ in length. As with the pressure analyses, this experiment is only a partial evaluation of the intended implant system, which would incorporate cuffs with electrodes and leads. It is assumed once again that they would have little influence on the infliction of neural damage as long as they are routed carefully at implantation.

1) Implantation Procedure: Two surgical procedures were performed on the cat, the median and ulnar nerves being implanted one week after the sciatic nerves. In each case endotracheal anesthesia was produced using a gaseous mixture of oxygen, nitrous oxide, and halothane. A heating pad was used to maintain the animal's normal body temperature. A salivation suppressant, IM atropine sulfate $(0.044 \mathrm{mg} / \mathrm{kg})$, and a broad-spectrum antibiotic, IM oxacillin sodium $(200 \mathrm{mg})$, also were administered. The implant sites were prepared for surgery. Using aseptic techniques, each nerve was exposed and measured with a sterile ruler ( $0.5 \mathrm{~mm}$ divisions). An appropriately sized cuff was chosen for implantation, rinsed in a sterile antibiotic solution of sodium cefazolin $(1 \mathrm{~g}$ in $250 \mathrm{ml}$ of lactated Ringer's solution) that was also used to irrigate the surgical sites, and wrapped around the nerve. The incisions were closed and the animal was closely monitored for several hours postoperatively.

2) Histological Procedure: Following the seven month maintenance period the animal was deeply anaesthetized with IM ketamine hydrochloride $(30 \mathrm{mg} / \mathrm{kg}$ ) and IV sodium pentobarbital $(0.5 \mathrm{mg}$ as needed), intubated, and maintained on a respirator. The chest was opened, intracardiac heparin sodium (1500 units) was administered to inhibit blood coagulation, and the animal was fixed and killed via aortic perfusion of low osmolarity fixatives: one liter of paraformaldehyde (1 percent in $25 \mathrm{mM}$ cacodylate buffer) followed by two liters of glutaraldehyde ( 3.5 percent in $25 \mathrm{mM}$ cacodylate buffer). Following perfusion, the nerves to be studied were cleanly dissected and further fixed in situ for 5 min by immersion in cold glutaraldehyde to ensure stabilization at natural physiological length. A segment of each nerve was excised $(4-5 \mathrm{~cm}$ in length), taking care to ensure that the implanted region was in the middle of this segment. For each nerve, the cuff's position was marked with suture and the cuff was removed. Each nerve segment was immersed in cold glutaraldehyde for one hour, rinsed in $25 \mathrm{mM}$ cacodylate buffer overnight, postfixed with osmium tetroxide, dehydrated in acetone series, and embedded in Spurr's low viscosity resin. Semithin transverse sections $(1 \mu \mathrm{m})$ were cut

\footnotetext{
${ }^{6}$ The desired implant CNR is determined before surgery. During surgery the diameter of the nerve to be implanted is measured with a sterile ruler. Since nerves often exhibit an elliptical cross section, the major and minor axes are measured as accurately as possible. An "average" nerve diameter is calculated from these figures. The cuff diameter necessary to achieve the previously determined CNR is then calculated and an appropriately sized cuff is chosen for implantation from those available. A visual inspection is performed before closing the surgical site so as to grossly verify that the cuff is snug, loose, or very loose upon the nerve. Alternate methods of measuring nerve diameter are being contemplated for future eperiments.
} 


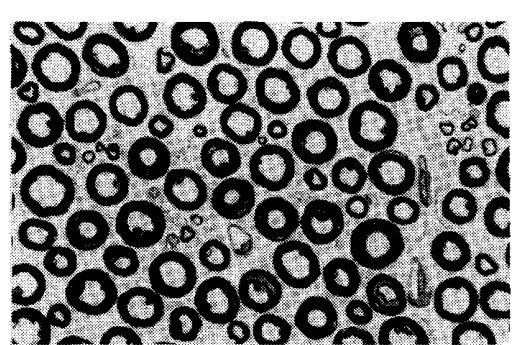

(a)

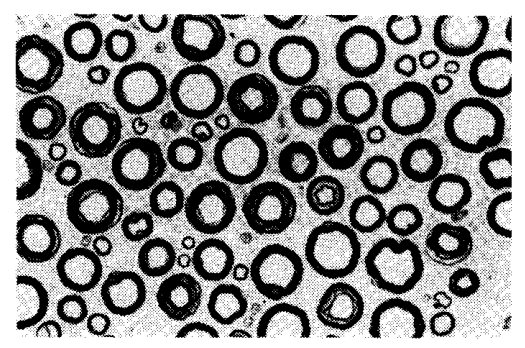

(b)

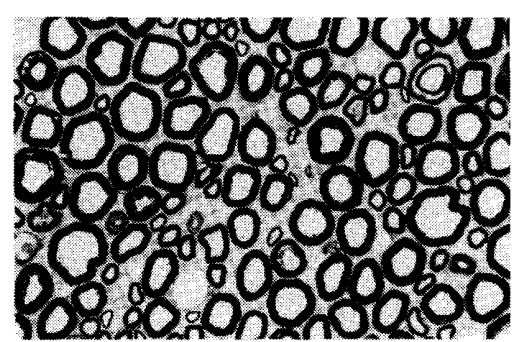

(c)

Fig. 9. Axons within the peripheral nerves that were implanted with snug spiral cuffs ( $C N R=0.8-0.9$ ) appeared normal at all three locations that were examined: proximal to the cuff (a), cuff level (b), and distal to the cuff $(\mathrm{c})$. (Magnification: $420 \times$ )

and stained with methylene blue borax for light microscopy. Each nerve was examined at three locations: proximal to the cuff, cuff level, and distal to the cuff.

\section{Results}

During the seven month maintenance period the cat typically was very active and was seen to exhibit normal gait. At the time of cuff explant, all implant sites were seen to be infiltrated with a thin layer of healthy, fibrous connective tissue. For each implanted nerve, the space between it and the cuff was fully infiltrated with this tissue. Each cuff also was enveloped by a thin capsule of connective tissue, ranging in thickness from 100 to $400 \mu \mathrm{m}$. In each case, the connective tissue capsule consisted of a matrix of collagen fibers infiltrated with fibroblasts and polymorphonuclear leukocytes. Monocytes could also be seen occasionally. These observations correlate with those for normal wound repair and were interpreted as a minimal foreign body response by the tissue.

Each of the implanted nerves ( 4 snug, 2 slightly loose) exhibited a normal appearance at each level that was examined (see Fig. 9). Axon profiles were observed to be comparable to those of nerves receiving no implants. Myelin thickness and axon distribution appeared normal. There appeared to be a slight increase in the quantity of intraneural connective tissue. There was no evidence of axonal degeneration, edema, or abnormal immunological reaction within the neural or connective tissues.

\section{Conclusions}

The spiral cuff potentially offers some important collective advantages that previous cuff designs do not. First, and most important, is that the spiral design apparently can be safely implanted at sizes which fit nerves very snugly. The only other configuration that shares this property is the HMRI helical electrode (which, strictly speaking, is not a cuff) [60].

Second, the self-sizing nature of the spiral design makes it easy to size snug cuffs on a nerve. A spiral cuff which naturally has a diameter slightly smaller than a nerve will automatically conform itself to the nerve. The HMRI helical electrode [60] again shares this particular property, but other cuff designs do not have the flexibility to automatically size themselves in this way.

Third, spiral cuff implantation is simple. While it is not terribly difficult to implant a split-cylinder cuff, the need to "seal" the cuff on the nerve trunk with suture is nonetheless eliminated with the spiral design.

Fourth, confinement of electric current within the cuff is maximized with the spiral design. Cuffs that must be sutured closed, or nerve electrodes that are not contained within a cuff (e.g., the HMRI open helix) may exhibit conduction paths such that current flow is not maximally confined within the cuff. The consequent "spillover" of current is undesirable when stimulating since it can give rise to the undesired activation of adjacent neural structures and because it implies that slightly higher stimulation levels may be necessary to accomplish a certain goal; it also is undesirable when recording ENG's because the signal-to-noise ratio will be reduced.

We have indicated that the most significant complication associated with the use of nerve cuff electrodes has been the unpredictable occurrence of mechanically-induced neural damage. Because of this, even the moderately successful split-cylinder cuffs have been abandoned in many cases, sometimes in favor of alternate electrode configurations (e.g., "half-cuff", electrodes [21] and epineural electrodes [36]). The spiral cuff design was devised so that passively-induced neural damage might be avoided, particularly when implanted snugly upon a nerve.

The results of our preliminary implant experiment are encouraging. The data support the theoretical prediction that spiral cuffs can be implanted safely when sized to fit peripheral nerve snugly. They also suggest that it may be possible to safely implant slightly loose spiral cuffs, though data from other experiments indicate that this is not always the case [35]. At this stage of evaluation, however, these results are not definitive, and we recognize the need for further experiments before the spiral cuff design 
can be contemplated for use in human subjects. Such experiments will be concerned with establishing with greater certainty the conditions under which the spiral cuff is safe for implantation. Future tests must also employ split-cylinder cuffs as well as spiral cuffs, so that comparative analyses can be made; and they must also incorporate entire cuff electrode assemblies, with electrodes and leads, in order to assess the assumption that carefully routed leads will not contribute significantly to the infliction of neural damage.

In conclusion, we believe that the spiral nerve cuff is a promising alternative to traditional designs. While evaluations of the spiral cuff are far from complete at this stage, the results of our initial evaluations and experiments suggest that this design has strong potential for application to neuroprosthetic technology in the future. Of particular interest is the ability to implant cuffs snugly. If additional data continue to support the finding that snug cuffs are safe for implantation, significant benefits become apparent, including: 1) for recording electroneurograms, the signal-to-noise ratio can be optimized [12][14], [46]-[48]; 2) for stimulation, the improved ability to create localized electric fields through the electrical manipulation of several electrodes makes feasible the possibility of selectively activating specific regions of the nerve and, hence, particular muscles or motor units [31], [49]; and 3) the stimulus magnitude required for neural activation can be minimized, conserving system power and decreasing the likelihood of electrically-induced neural damage [32].

In addition to these specific benefits of snug cuffs, the routine ability to reliably and safely implant nerve cuffs of any size would permit the full exploitation of their'general advantages delineated at the outset of this paper. Newer electrical stimulation techniques for blocking neural conduction [50], [54]-[56] and activating motor units in their natural recruitment order [18], [19] offer great promise for future neuroprosthetic applications, but they can only be realized through the use of nerve cuff electrodes. Because of this, we encourage the implementation of new, comprehensive studies that focus on the evaluation of the passive safety of any and all practical nerve cuff electrode designs.

\section{ACKNOWLEDGMENTS}

We wish to thank Dr. U. Roessmann, Dr. P. Gambetti, and Dr. A. Morandi of the University Hospitals of Cleveland (Department of Pathology, Neuropathology Division) for their help in evaluating tissue integrity; our histotechnician Y. Dietz; N. Caris for assisting during surgery; A. S. Ferguson for software support; and C. L. Van Doren for his comments on the manuscript.

\section{REFERENCES}

[1] AAMI American National Standard for Implantable Peripheral Nerve Stimulators (ANSI/AAMI NS15-1984). Arlington, VA: Association for the Advancement of Medical Instrumentation, 1984.

[2] R. E. Avery and J. S. Wepsic, "Implantable electrodes for the stimulation of the sciatic nerve," U.S. Patent 3738368 June 12, 1973.
[3] R. E. Avery, "Implantable nerve stimulation electrode," U.S. Patent 3774618 , Nov. $27,1973$.

[4] R. B. Banzett, " Implantable electrode pair for recording from intact small nerves," IEEE Trans. Biomed. Eng., vol. BME-27, pp. 53-54, 1980.

[5] F. C. Barone, M. J. Wayner, H. U. Aguilar-Baturoni, and R. Guevara-Aguilar, "A bipolar electrode for peripheral nerve stimulation," Brain Res. Bull., vol. 4, pp. 421-422, 1979.

[6] A. Blake, Design of Curved Members for Machines. New York: Industrial Press, 1966, ch. 10, pp. 152-154.

[7] A. P. Boresi and O. M. Sidebottom, Advanced Mechanics of Materials, Fourth Edition. New York: Wiley, 1985, ch. 11, p. 500

[8] R. S. Breederveld and G. Zilvold, "Electrode implantation and electrostimulation in rabbits," Arch. Phys. Med. Rehabil., vol. 60, pp. $162-164,1979$

[9] G. S. Brindley, C. E. Polkey, and D. N. Rushton, "Electrical splinting of the knee in paraplegia," Paraplegia, vol. 16, pp. 428-435, 1978-1979.

[10] F. W. Clippinger, R. Avery, and B. R. Titus, "A sensory feedback system for an upper-limb amputation prosthesis," Bull. Proc. Res., vol. BPR 10-22, pp. 247-258, 1974.

[11] P. E. Crago, P. H. Peckham, and G. B. Thrope, "Modulation of muscle force by recruitment during intramuscular stimulation," IEEE Trans. Biomed. Eng., vol. BME-27, pp. 679-684, 1980.

[12] L. A. Davis, T. Gordon, J. A. Hoffer, J. Jhamandas, and R. B. Stein, "Compound action potentials recorded from mammalian peripheral nerves following ligation or resuturing," $J$. Physiol., vol. 285 , pp. $543-559,1978$.

[13] C. J. DeLuca and L. D. Gilmore, "Voluntary nerve signals from severed mammalian nerves: Long-term recordings," Science, vol. 191, pp. 193-195, 1976.

[14] C. J. DeLuca, L. D. Gilmore, L. J. Bloom, S. J. Thomson, A. L. Cudworth, and M. J. Glimcher, "Long-term neuroelectric signal recording from severed nerves," IEEE Trans. Biomed. Eng., vol. BME29 , pp. 393-402, 1982.

[15] C. Dubkin, "A constant-contact stimulating electrode for nerves," J. Appl. Physiol., vol. 28, p. 350, 1970.

[16] T. B. Ducker and G. J. Hayes, "Experimental improvements in the use of silastic cuff for peripheral nerve repair," J. Neurosurg., vol. 28 , pp. 582-587, 1968 .

[17] D. J. Edell, "A peripheral nerve information transducer for amputees: Long-term multichannel recordings from rabbit peripheral nerves," IEEE Trans. Biomed. Eng., vol. BME-33, pp. 203-214, 1986.

[18] Z. P. Fang and J. T. Mortimer, "A method for attaining natural recruitment order in artificially activated muscles," in Proc. 9th Annu. Conf. IEEE Eng. Med. Biol. Soc., New York, 1987, pp. 657-658.

[19] - "Selective activation of small motor axons by spiral cuff electrode and quasi-trapezoidal stimulus pulses,"'Soc. Neurosci. Abstr., vol. 13, p. 1698, 1987.

[20] W. W. L. Glenn, J. F. Hogan, J. S. O. Lake, T. E. Ciesielski, M L. Phelps and R. Rowedder, "Ventilatory support by pacing of the conditioned diaphragm in quadraplegia," N.E.J. Med, vol. 310 , pp. $1150-1155,1984$

[21] W. W. L. Glenn and M. L. Phelps, "Diaphragm pacing by electrical stimulation of the phrenic nerve," Neurosurg., vol. 17, pp. 974-984, 1985.

[22] P. A. Grandjean and J. T. Mortimer, "Recruitment properties of monopolar and bipolar epimysial electrodes," Ann. Biomed. Eng., vol. 14, pp. 53-66, 1986.

[23] N. R. Hagfors, "Implantable electrode," U.S. Patent 3654 933, Apr. 11,1972 .

[24] C. Julien and S. Rossignol, "Electroneurographic recordings with polymer cuff electrodes in paralyzed cats," J. Neurosci. Meth., vol. 5 , pp. 267-272, 1982.

[25] J. H. Kim, E. E. Maneulidis, W. W. L. Glenn, and T. Kaneyuki, "Diaphram pacing-Histopathological changes in the phrenic nerve following long-term electrical stimulation," J. Thor. Cardiovasc. Surg., vol. 72, pp. 602-608, 1976.

[26] J. H. Kim, E. E. Manuelidis, W. W. L. Glenn, Y. Fukuda, D. S, Cole, and J. F. Hogan, "Light and electron microscopic studies of phrenic nerves after long-term electrical stimulation," J. Neurosurg., vol. 58, pp. 84-91, 1983.

[27] L. P. McCarty, "Nerve electrode apparatus," U.S. Patent 3157181 , Nov. 17, 1964.

[28] L. P. McCarty, "A stimulating electrode for nerves," J. Appl. Physiol., vol. 20, p. 542, 1965. 
[29] D. R. McNeal, R. Waters, and J. Reswick, "Experience with implanted electrodes," Neurosurg., vol. 1, pp. 228-229, 1977.

[30] - "Experience with implanted electrodes at Rancho Los Amigos Hospital,"' Appl. Neurophysiol., vol. 40, pp. 235-239, 1977/1978.

[31] D. R. McNeal and B. R. Bowman, "Selective activation of muscles using peripheral nerve electrodes," Med. Biol. Eng. Comput., vol. 23, pp. 249-253, 1985.

[32] J. T. Mortimer, "Motor prostheses" in Handbook of Physiology, Section 1: The Nervous System-Vol. II Motor Control, Part 1, J. M, Brookhart, V. B. Mountcastle, V. B. Brooks, S. R. Geiger, Eds. Bethesda, MD: Amer. Physiol. Soc., 1981, ch. 5, pp. 155-187.

[33] G. G. Naples, J. D. Sweeney, and J. T. Mortimer, "Implantable cuff, method of manufacture, and method of installation," U.S. Patent 4602624 , July $29,1986$.

[34] G. G. Naples, "An implantable spiral cuff electrode for electrical stimulation of peripheral nerve," M.S. thesis, Case Western Reserve Univ., Cleveland, OH, May 1987.

[35] G. G. Naples, J. T. Mortimer, and T. G. H. Yuen, "Biocompatibility of peripheral nerve electrodes and materials," in Neural Prostheses: Fundamental Studies, W. F. Agnew and D. B. McCreery, Eds. En glewood Cliffs, NJ: Prentice-Hall, to be published.

[36] B. S. Nashold, J. L. Goldner, J. B. Mullen, and D. S. Bright, " Longterm pain control by direct peripheral nerve stimulation," "J. Bone Joint Sug., vol. 64A, pp. 1-10, 1982.

[37] K. D. Nielson, C. Watts, and W. K. Clark, "Peripheral nerve injury from implantation of chronic stimulating electrodes for pain control,", Surg. Neurol., vol. 5, pp. 51-53, 1976.

[38] I. Ninomiya, Y. Yonezawa, and M. F. Wilson, "Implantable electrode for recording nerve signals in awake animals," J. Appl. Phys., vol. 41, pp. 111-114, 1976.

[39] J. A. Picaza, B. W. Cannon, S. E. Hunter, A. S. Boyd, J. Guma, and D. Maurer, "Pain suppression by peripheral nerve stimulationPart II, observations with implanted devices," Surg. Neurol., vol. 4, pp. 115-126, 1975.

[40] J. A. Picaza, S. E. Hunter, and B. W. Cannon, "Pain suppression by peripheral nerve stimulation-Chronic effects of implanted devices," Appl. Neurophysiol., vol. 40, pp. 223-234, 1977/1978.

[41] B. Rydevik, G. Lundborg, and U. Bagge, "Effects of graded compression on intraneural blood flow-An in vivo study on rabbit tibial nerve," J. Hand Surg., vol. 6, pp. 3-12, 1981

[42] T. Sakaguchi, A. Warashina, and A. Niijima, "Production of elastic electrodes for nerve stimulation," Pflugers Arch., vol. 380, p. 283, 1979.

[43] J. F. Sauter, H. R. Berthoud, and B. Jeanrenaud, " A simple electrode for intact nerve stimulation and/or recording in semi-chronic rats," Pflugers Arch., vol. 397, pp. 68-69, 1983.

[44] R. A. Schmidt, H. Bruschini, and E. A. Tanagho, "Feasibility of inducing micturition through chronic stimulation of sacral roots," Urol., vol. 12, pp. 471-477, 1978.

[45] S. I. Schwartz, R. C. Wingrove, and J. A. Anderson, "Implantable electrode for nerve stimulation," U.S. Patent Re. 26810 Mar. 3, 1970. Original U.S. Patent 3421511 , Jan. 14, 1969

[46] R. B. Stein, D. Charles, L. Davis, J. Jhamandas, A. Mannard, and T. R. Nichols, "Principles underlying new methods for chronic neural recording," Canad. J. Neurol. Sci., vol. 2, pp. 235-244, 1975.

[47] R. B. Stein, T. R. Nichols, J. Jhamandas, L. Davis, and D. Charles, "Stable long-term recordings from cat peripheral nerves," Brain Res., vol. 128, pp. 21-38, 1977

[48] R. B. Stein, D. Charles, T. Gordon, J. Hoffer, and J. Jhamandas, "Impedance properties of metal electrodes for chronic recording from mammalian nerves," IEEE Trans. Biomed. Eng., vol. BME-25, pp. 532-536, 1978.

[49] J. D. Sweeney, D. A. Ksienski, and J. T. Mortimer, "A nerve cuff technique for selective motor control," submitted for presentation at the 10th Annu. Int. Conf. IEEE Eng. Med. Biol. Soc., New Orleans, LA, 1988.

[50] J. D. Sweeney and J. T. Mortimer, "An asymmetric two electrode cuff for generation of unidirectionally propagated action potentials," IEEE Trans. Biomed. Eng., vol. BME-33, pp. 541-549, 1986.

[51] E. A. Tanagho and R. A. Schmidt, "Bladder pacemaker: Scientific basis and clinical future,' Urol., vol. 20, pp. 614-619, 1982.

[52] R. L. Testerman, N. R. Hagfors, and S. I. Schwartz, "Design and evaluation of nerve stimulating electrodes," Med. Res. Eng., vol. 10 pp. 6-11, 1971.

[53] R. L. Testerman, "Shielded recording electrode system," U.S. Patent 4341221 , July $27,1982$.

[54] I. J. Ungar, J. T. Mortimer, and J. D. Sweeney, "Generation of unidirectionally propagating action potentials using a monopolar electrode cuff,"' Ann. Biomed. Eng., vol. 14, pp. 437-450, 1986.

[55] C. van den Honert and J. T. Mortimer, "A technique for collision block of peripheral nerve: Single stimulus analysis," IEEE Trans. Biomed. Eng., vol. BME-28, pp. 373-378, 1981.

[56] — , "A technique for collision block of peripheral nerve: Frequency dependence," IEEE Trans. Biomed. Eng., vol. BME-28, pp. 379$382,1981$.

[57] R. L. Waters, D. McNeal, and J. Perry, "'Experimental correction of footdrop by electrical stimulation of the peroneal nerve," $J$. Bone Joint Surg., vol. 57A, pp. 1047-1054, 1975.

[58] R. L. Waters, D. R. McNeal, and J. Tasto, "Peroneal nerve conduction velocity after chronic electrical stimulation," Arch. Phys. Med. Rehabil., vol. 56, pp. 240-243, 1975.

[59] R. L. Waters, D. R. McNeal, W. Faloon, and B. Clifford, "Functional electrical stimulation of the peroneal nerve for hemiplegia: Long-term clinical follow-up," J. Bone Joint Surg., vol. 67-A, pp. $792-793,1985$.

[60] T. G. H. Yuen, W. F. Agnew, and L. A. Bullara, "Histopathological evaluation of dog sacral nerve after chronic electrical stimulation for micturition," Neurosurg., vol. 14, pp. 449-455, 1984.

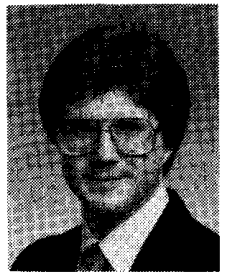

Gregory G. Naples (S'82-M'87) was born in Cleveland, $\mathrm{OH}$, in 1961. He received the B.S. degree in electrical engineering from the University of Notre Dame, Notre Dame, IN, in 1983, and the M.S. degree in biomedical engineering from Case Western Reserve University, Cleveland, $\mathrm{OH}$, in 1987.

$\mathrm{He}$ is currently a Biomedical Engineer at the Case Western Reserve University Rehabilitation Engineering Center in the Department of Orthopaedics and Rehabilitation, Cleveland Metropolitan General/Highland View Hospital. His interests are in applied neural control, rehabilitation engineering, business administration, and regulatory affairs as related to medical devices.

Mr. Naples is an associate member of the Biomedical Engineering Society, and a member of Tau Beta Pi, Eta Kappa $\mathrm{Nu}$, and the Regulatory Affairs Professionals Society.

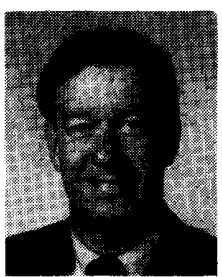

J. Thomas Mortimer received the B.S.E.E. degree from Texas Technological College, Lubbock, TX, in 1964, and the Ph.D. degree from Case Western Reserve University, Cleveland, $\mathrm{OH}$, in 1968 .

From 1968 to 1969 he was a Research Associate at Chalmers Institute of Technology, Gothenberg, Sweden. From 1977 to 1978 he was a Visiting Professor at the Institute for Biocybernetics, University of Karlsruhe, Karlsruhe, Germany. Currently, he is Professor of Biomedical Engineering, and Director of the Applied Neural Control Laboratory, Case Western Reserve University. His past and present research, in areas involving electrical stimulation, includes pain suppression, restoration of hand function in quadriplegic patients, scoliosis correction, electrophrenic respiration, and neural block.

Dr. Mortimer was the recipient of the Humboldt Preis (Senior U.S. Scientist Award), Alexander von Humboldt Foundation, Federal Republic of Germany. He is a member of Tau Beta Pi, Eta Kappa Nu, and Sigma Xi. 


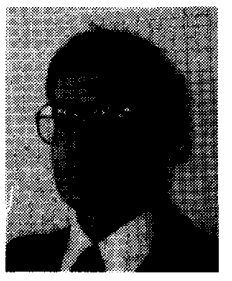

Avram Scheiner was born in Pittsburgh, $\mathrm{PA}$, in 1958. He received the B.S. degree in mechanical engineering from Carnegie-Mellon University, Pittsburgh, PA, in 1980, and the M.S. degree in biomedical engineering from Case Western Reserve University, Cleveland, $\mathrm{OH}$, in 1988.

$\mathrm{He}$ is currently an Engineer at the Veterans Administration Medical Center, Cleveland, $\mathrm{OH}$.

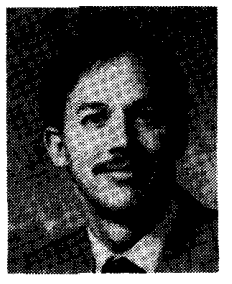

James D. Sweeney was born in Pittsburgh, PA in 1957. He received the Sc.B. degree in biomedical engineering from Brown University, Providence, RI, in 1979, and the M.S. and Ph.D. de

grees in biomedical engineering from Case Western Reserve University, Cleveland, $\mathrm{OH}$, in 1983 and 1988 , respectively.

During this research, he was a Predoctoral Fellow at Case Western Reserve University under sponsorship of the Paralyzed Veterans of America. He is currently a Research Associate in the Department of Biomedical Engineering at Case Western Reserve University. His research interests are in applied neural control, neurophysiology, and neural modeling, especially as related to the fields of neural prostheses and rehabilitation engineering. 